\title{
Four-boson system with short-range interactions
}

\author{
L. Platter* \\ Forschungszentrum Jülich, Institut für Kernphysik, D-52425 Jülich, Germany \\ and Helmholtz-Institut für Strahlen-und Kernphysik (Theorie), Universität Bonn, Nussallee 14-16, D-53115 Bonn, Germany
}

H.-W. Hammer ${ }^{\dagger}$

Institute for Nuclear Theory, University of Washington, Seattle, Washington 98195, USA

Ulf-G. Meißner

Helmholtz-Institut für Strahlen-und Kernphysik (Theorie), Universität Bonn, Nussallee 14-16, D-53115 Bonn, Germany

and Forschungszentrum Jülich, Institut für Kernphysik, D-52425 Jülich, Germany

(Received 15 April 2004; revised manuscript received 2 July 2004; published 5 November 2004)

\begin{abstract}
We consider the nonrelativistic four-boson system with short-range forces and large scattering length in an effective quantum mechanics approach. We construct the effective interaction potential at leading order in the large scattering length and compute the four-body binding energies using the Yakubovsky equations. Cutoff independence of the four-body binding energies does not require the introduction of a four-body force. This suggests that two- and three-body interactions are sufficient to renormalize the four-body system. We apply the equations to ${ }^{4} \mathrm{He}$ atoms and calculate the binding energy of the ${ }^{4} \mathrm{He}$ tetramer. We observe a correlation between the trimer and tetramer binding energies similar to the Tjon line in nuclear physics. Over the range of binding energies relevant to ${ }^{4} \mathrm{He}$ atoms, the correlation is approximately linear.
\end{abstract}

DOI: 10.1103/PhysRevA.70.052101

PACS number(s): 03.65.Ge, 36.40.-c, 21.45.+v

\section{INTRODUCTION}

Effective theories are ideally suited to describe the lowenergy properties of physical systems in a modelindependent way. They can be applied to any system that has a separation of scales, which can be a fundamental property of the underlying theory or simply a kinematical suppression. The long-distance degrees of freedom must be included dynamically in the effective theory, while short-distance physics enters only through the values of a few coupling constants, often called low-energy constants. Effective theories are widely used in many areas of physics. Recently, a considerable effort was devoted to applying effective field theories in nuclear and atomic physics. For overviews of these programs, see, e.g., Refs. [1-4]. If there is no exchange of massless particles, any interaction will appear short ranged at sufficiently low energy. One can then use a very general effective theory with short-range interactions only to describe the universal low-energy properties of the system. Such a theory can be applied to a wide range of systems from nuclear and particle physics to atomic and molecular physics.

Most previous work in this area was done using a nonrelativistic effective-field theory (EFT) with contact interactions. Particularly interesting are few-boson systems with large scattering length. They are characterized by an unnaturally large two-body scattering length $a$ which is much larger than the typical low-energy length scale $l$ given by the range of the interaction. Such systems display a number of inter-

\footnotetext{
*Electronic address: 1.platter@fz-juelich.de

†Electronic address: hammer@phys.washington.edu

${ }^{\ddagger}$ Electronic address: meissner@itkp.uni-bonn.de
}

esting effects and universal properties that are independent of the details of the interaction at short distances of order $l$ : If $a>0$, e.g., there is a shallow two-body bound state with binding energy $B_{2}=\hbar^{2} /\left(M a^{2}\right)+\mathcal{O}(l / a)$, where $M$ is the mass of the particles. Low-energy observables can generally be described in a controlled expansion in $l /|a|$. In the two-boson system, the effective theory reproduces the effective range expansion (cf. Refs. [5,6]) but the structure of the threeboson system with large scattering length is richer. In Refs. $[7,8]$, it was found that both two- and three-body contact interactions are required at leading order for the consistent renormalization of the three-body system. Interestingly, the renormalization-group behavior of the three-body interaction is governed by an ultraviolet limit cycle. This implies that at leading order in $l /|a|$, the properties of the three-boson system with large scattering length are not determined by twobody data alone and one piece of three-body information (such as a three-body binding energy) is required as well. In the EFT, this information can conveniently be parametrized by the three-body parameter $\Lambda_{*}$ introduced in Refs. [7,8]. These general findings confirm and extend previous work by Efimov who derived many general features of the three-body problem with large scattering length $[9,10]$.

While the EFT formulation has been very successful, it is not the only possible formulation of an effective theory for this problem. Lepage has advocated the framework of nonrelativistic quantum mechanics with an effective interaction potential [11]. The contact operators in the field theory are the replaced by an "effective potential" built from smeared out $\delta$-function potentials and derivatives thereof. In the case $a>0$, this approach has been applied to the three-boson system by Wilson [12] and Mohr [13]. They confirmed the results of Refs. $[7,8]$ and were able to calculate the binding 
energies for the three-boson system to extremely high accuracy.

In this paper, we consider the four-body system with short-range interactions. The four-body problem has previously been studied in a variety of approaches. Early studies include the Yakubovsky equations for local potentials using the Hilbert-Schmidt expansion [14], the Schrödinger equation with separable two-body potentials [15], and fieldtheoretical models with separable expansions of the threebody $T$ matrix [16]. The four-body problem of ${ }^{4} \mathrm{He}$ atoms was investigated by Nakaichi-Maeda and Lim using the Yakubovsky equations with a unitary pole approximation for the $S$-wave (2)+(2) and (3)+(1) subamplitudes [17]. For a review of these and other early studies see, e.g., Refs. $[18,19]$. For an overview of recent calculations for the fourbody system of ${ }^{4} \mathrm{He}$ atoms, see Refs. [21,22]. A general review of theoretical studies of small ${ }^{4} \mathrm{He}$ clusters can be found in Ref. [23]. In Ref. [24], a benchmark calculation comparing various modern calculational approaches to the nuclear four-body problem was carried out.

The purpose of this paper is to study the four-boson system with short-range interactions and large scattering length in an effective theory. We will work at leading order in $l /|a|$ and use the framework of nonrelativistic quantum mechanics to construct an effective interaction potential. This approach has the advantage that one can immediately start from the well-known Yakubovsky equations for the four-body system [25]. The four-boson binding energies are obtained by solving the Yakubovsky equations for the effective interaction potential. The solution of the four-boson problem in effective theory is important in several respects:

First, it can immediately be applied to the atomic problem of ${ }^{4} \mathrm{He}$ atoms and is a first step towards the four-body problem in nuclear physics which is complicated by spin and isospin. The scattering length of ${ }^{4} \mathrm{He}$ atoms $a \approx 100 \AA$ is much larger than its effective range $r_{e} \approx 7 \AA$ which can be taken as an estimate of the natural low-energy length scale $l$. ${ }^{4} \mathrm{He}$ atoms are therefore an ideal application for our theory. The three-body system of ${ }^{4} \mathrm{He}$ atoms has been investigated in Refs. $[8,26,27]$ using effective-field theory. While the universal properties of the three-body system of ${ }^{4} \mathrm{He}$ atoms were discussed in Ref. [26], this has not been done for the fourbody system.

Second, the renormalization of the four-body system in an effective theory is an open question. It is clear that lowenergy four-body observables must depend on a two-body parameter and a three-body parameter. However, it is not known whether a four-body parameter is also required to calculate low-energy four-body observables up to corrections suppressed by $l /|a|$.

The theoretical situation concerning this question appears confusing. On the one hand, there is a renormalization argument for $\delta$-function pair potentials that indicates that a new four-body parameter is required to calculate four-body binding energies [28]. On the other hand, Amado and Greenwood have evaluated the trace of the four-body kernel and concluded that the Efimov effect is absent in the four-body system [29]. This result suggests that a four-body parameter should not be necessary at leading order in $l /|a|$. There is some circumstantial evidence in favor of the latter possibility from the four-body problem in nuclear physics. There is a correlation called the "Tjon line" between the binding energy $B_{t}$ of the triton and the binding energy $B_{\alpha}$ of the $\alpha$ particle [14]. Calculations of these binding energies using modern phenomenological nucleon-nucleon interaction potentials give results that underestimate both binding energies but cluster along a line in the $B_{t}-B_{\alpha}$ plane. By adding a threebody potential whose strength is adjusted to get the correct value for $B_{t}$, one also gets an accurate result for $B_{\alpha}$ (cf. Ref. [30]). This conclusion also holds for chiral nuclear potentials derived from an effective-field theory with explicit pions [31]. The aim of the present work is to study the question about the requirement of the four-body interaction by an explicit calculation in the controlled environment of an effective theory.

The paper is organized as follows. In Sec. II, we will review the description of the two- and three-body bound state problem in effective theory and extend this framework to the four-body bound state problem. In Sec. III, we will discuss the renormalization and present numerical results for the case of ${ }^{4} \mathrm{He}$ atoms. Finally, we close with a summary and outlook in Sec. IV.

\section{FEW-BODY BOUND STATE EQUATIONS IN EFFECTIVE THEORY}

The effective low-energy interaction potential generated by a nonrelativistic EFT with short-range interactions can be written down in a momentum expansion. In the two-body $S$-wave sector, it takes the general form

$$
\left\langle\mathbf{k}^{\prime}|V| \mathbf{k}\right\rangle=\lambda_{2}+\lambda_{2,2}\left(k^{2}+k^{\prime 2}\right) / 2+\ldots,
$$

where $\mathbf{k}$ and $\mathbf{k}^{\prime}$ are the relative three-momenta of the incoming and outgoing particles, respectively. Because of Galilean invariance, the interaction can only depend on the relative momenta. Similar expressions can be derived for three- and higher-body interactions. The exact form of the potential depends on the specific regularization scheme used. The lowenergy observables, however, are independent of the regularization scheme (up to higher-order corrections) and one can choose a convenient scheme for practical calculations. ${ }^{1}$

In a momentum cutoff scheme, the potential in Eq. (1) can be regularized by multiplying with a Gaussian regulator function, $\exp \left[-\left(k^{2}+k^{\prime 2}\right) / \Lambda^{2}\right]$, with the cutoff parameter $\Lambda$. This factor strongly suppresses high-momentum modes in the region $k, k^{\prime} \gtrsim \Lambda$ where the effective potential is not valid. The cutoff dependence of the coefficients $\lambda_{2}(\Lambda), \lambda_{2,2}(\Lambda), \ldots$ is determined by the requirement that low-energy observables are independent of $\Lambda$. Of course, the expansion in Eq. (1) is only useful in conjunction with a power counting scheme that determines the relative importance of the various terms at low energy. In the case of large scattering length $a$, the leading order is given by the $\lambda_{2}$ term which must be iterated to all orders, while the other terms give rise to

\footnotetext{
${ }^{1}$ For a comparison of different regularization schemes in the nuclear two-body problem and chiral perturbation theory, see Refs. $[32,33]$, respectively.
} 
higher-order corrections that can be included perturbatively $[5,6]$. In this paper, we will work to leading order in the large scattering length $a$ and include only the $\lambda_{2}$ term.

In the three-body system, a momentum-independent three-body interaction term $\lambda_{3}$ must be included together with $\lambda_{2}$ already at leading order $[7,8]$. Without this threebody interaction low-energy observables show a strong cutoff dependence and the system cannot be renormalized. Effective range effects and other higher-order corrections can be included as well [34-36].

The power counting for the four-body system has not been formulated yet. In order to see whether the minimal set of interactions $\lambda_{2}$ and $\lambda_{3}$ is sufficient, we will calculate the bound states in the four-body system and study their cutoff dependence. We will use a momentum cutoff regularization scheme as described above. A strong cutoff dependence of the binding energies would indicate that a four-body interaction term is required. If the four-body binding energies are stable under variations of the cutoff, this would suggest that the four-body interaction is a subleading effect.

In order to set up our conventions and formalism, we will first review the bound state equations for the two- and threebody system and then set up the four-body equations.

\section{A. The two-body sector}

We write the leading order two-body effective potential in momentum space as

$$
\langle\mathbf{p}|V| \mathbf{q}\rangle=\langle\mathbf{p} \mid g\rangle \lambda_{2}\langle g \mid \mathbf{q}\rangle,
$$

where $\lambda_{2}$ denotes the two-body coupling constant and $\mathbf{q}(\mathbf{p})$ are the relative three-momenta in the incoming (outgoing) channel. The regulator functions

$$
\langle\mathbf{p} \mid g\rangle \equiv g(p)=\exp \left(-p^{2} / \Lambda^{2}\right)
$$

suppress the contribution from high momentum states. In the few-body literature, they are often called "form factors."

Our normalization for plane-wave and spherical-wave states is

$$
\begin{aligned}
\left\langle\mathbf{p} \mid \mathbf{p}^{\prime}\right\rangle & =\delta^{(3)}\left(\mathbf{p}-\mathbf{p}^{\prime}\right), \\
\left\langle p l m \mid p^{\prime} l^{\prime} m^{\prime}\right\rangle & =\frac{\delta\left(p-p^{\prime}\right)}{p p^{\prime}} \delta_{l l^{\prime}} \delta_{m m^{\prime}},
\end{aligned}
$$

and the $S$-wave projection of the plane wave state with momentum $\mathbf{p}$ is

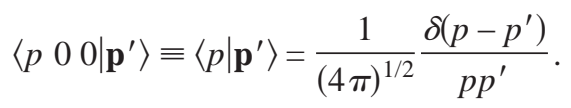

For convenience, we will work in units where the mass $M$ of the bosons and Planck's constant $\hbar$ are set to unity: $M=\hbar$ $=1$.

The interaction (2) is separable and the LippmannSchwinger equation for the two-body problem can be solved analytically. The two-body $t$ matrix can be written as [37]

$$
t(E)=|g\rangle \tau(E)\langle g|,
$$

where $E$ denotes the total energy. The two-body propagator $\tau(E)$ is then given by

$$
\tau(E)=\left[\frac{1}{\lambda_{2}}-4 \pi \int_{0}^{\infty} d q q^{2} \frac{g(q)^{2}}{E-q^{2}}\right]^{-1} .
$$

A two-body bound-state appears as a simple pole in the two-body propagator $\tau$ at energy $E=-B_{2}$. Thus the two-body coupling constant $\lambda_{2}\left(B_{2}, \Lambda\right)$ can be fixed from the two-body binding energy $B_{2}$, which is directly related to the scattering length by $a=1 / \sqrt{B_{2}}$ at leading order in $l /|a|$. The integrals appearing in the propagator can be expressed through the complementary error function

$$
\operatorname{erfc}(x)=1-\frac{2}{\sqrt{\pi}} \int_{0}^{x} e^{-t^{2}} d t
$$

and for $E<0$, we obtain for the inverse propagator:

$$
\begin{aligned}
\tau(E)^{-1}= & 2 \pi^{2}\left[\sqrt{B_{2}} \exp \left(\frac{2 B_{2}}{\Lambda^{2}}\right) \operatorname{erfc}\left(\frac{\sqrt{2 B_{2}}}{\Lambda}\right)\right. \\
& \left.-\sqrt{-E} \exp \left(\frac{-2 E}{\Lambda^{2}}\right) \operatorname{erfc}\left(\frac{\sqrt{-2 E}}{\Lambda}\right)\right] .
\end{aligned}
$$

A similar expression for the propagator can be obtained for positive energies by adding a small imaginary part to $E$, but will not be required for our purposes.

\section{B. The three-body sector}

The low-energy properties of the three-body system for a given effective potential can be obtained by solving the Faddeev equations [38]. Faddeev's idea was to decompose the full three-body wave function $\Psi$ into so-called "Faddeev components" in order to avoid the problem of disconnected contributions in the three-body scattering problem. For the three-body problem with two- and three-body interactions, the full wave function can be decomposed into four components [39]: one for each two-body subcluster and one for the three-body cluster. ${ }^{2}$ For identical bosons, the three-body wave function is fully symmetric under exchange of particles and the Faddeev equations simplify considerably. In this case, one only needs to solve equations involving one of the two-body Faddeev components and the three-body component. The two remaining two-body components can be obtained by permutations of particles. For more details on the Faddeev equations, we refer the reader to the literature $[40,37]$.

We follow Glöckle and Meier [39] and decompose the full three-body wave function as

$$
\Psi=(1+P) \psi+\psi_{3}, \quad \text { where } \quad P=P_{13} P_{23}+P_{12} P_{23}
$$

is a permutation operator that generates the two not explicitly included Faddeev components from $\psi$. The operator $P_{i j}$ simply permutes particles $i$ and $j$. The Faddeev equations for $\psi$ and $\psi_{3}$ in operator form are then

$$
\psi=G_{0} t P \psi+G_{0} t \psi_{3},
$$

\footnotetext{
${ }^{2}$ Note, however, that other decompositions involving only three Faddeev components are possible as well.
} 


$$
\psi_{3}=G_{0} t_{3}(1+P) \psi,
$$

where $G_{0}$ denotes the free three-particle propagator. $t$ is the two-body $t$ matrix for the two-body subsystem described by the component $\psi \cdot t_{3}$ is the three-body $t$ matrix defined by the solution of the three-body Lippmann-Schwinger equation with the leading order three-body effective interaction

$$
V_{3}=|\xi\rangle \lambda_{3}\langle\xi|,
$$

only. Since $V_{3}$ is separable, we can solve for $t_{3}$ exactly and obtain

$$
t_{3}(E)=|\xi\rangle \tau_{3}(E)\langle\xi|, \quad \text { where } \quad \tau_{3}(E)=\left[\frac{1}{\lambda_{3}}-\left\langle\xi\left|G_{0}\right| \xi\right\rangle\right]^{-1} .
$$

The three-body regulator function $|\xi\rangle$ will be specified later. Note that $t_{3}$ is only a technical construct that is generally cutoff dependent and not observable. The physical threebody $t$ matrix always includes both two- and three-body forces. Since we are interested only in the binding energies and not in the wave functions, we can eliminate the component $\psi_{3}$ and obtain

$$
\psi=G_{0} t P \psi+G_{0} t G_{0} t_{3}(1+P) \psi .
$$

The component $\psi_{3}$ can easily be recovered by using the second line of Eq. (11).

We now derive an explicit representation of Eq. (14) in momentum space. We will illustrate this procedure by showing some details for the first term on the right-hand side of Eq. (14). The extension to include the second term containing three-body interaction is straightforward and we will only quote the final result. Furthermore, in order to understand the renormalization of the three-body problem it is instructive to consider the case without a three-body force first. The natural Jacobi momenta are given by

$$
\mathbf{u}_{1}=\frac{1}{2}\left(\mathbf{k}_{1}-\mathbf{k}_{2}\right), \quad \mathbf{u}_{2}=\frac{2}{3}\left[\mathbf{k}_{3}-\frac{1}{2}\left(\mathbf{k}_{1}+\mathbf{k}_{2}\right)\right] .
$$

Here and in the following sections, we will only take $S$ waves into account. Hence we can project all operators accordingly and define the $S$-wave projection operator

$$
\int d u_{1} u_{1}^{2} d u_{2} u_{2}^{2}\left|u_{1} u_{2}\right\rangle\left\langle u_{1} u_{2}\left|\equiv \int \mathcal{D} u u^{2}\right| u_{1} u_{2}\right\rangle\left\langle u_{1} u_{2}\right| .
$$

Using the definition $\left\langle u_{1} u_{2} \mid \psi\right\rangle \equiv \psi\left(u_{1}, u_{2}\right)$, we can write the Faddeev equation in momentum space as

$$
\begin{aligned}
\psi\left(u_{1}, u_{2}\right)= & \left\langle u_{1} u_{2}\left|G_{0} t P\right| \psi\right\rangle \\
= & 4 \pi G_{0}\left(u_{1}, u_{2}\right) g\left(u_{1}\right) \int \mathcal{D} u^{\prime} u^{\prime 2} \int \mathcal{D} u^{\prime \prime} u^{\prime \prime 2} g\left(u_{1}^{\prime}\right) \\
& \times \tau\left(E-\frac{3}{4} u_{2}^{\prime 2}\right) \frac{\delta\left(u_{2}-u_{2}^{\prime}\right)}{u_{2}^{\prime 2}}\left\langle u_{1}^{\prime} u_{2}^{\prime}|P| u_{1}^{\prime \prime} u_{2}^{\prime \prime}\right\rangle\left\langle u_{1}^{\prime \prime} u_{2}^{\prime \prime} \mid \psi\right\rangle,
\end{aligned}
$$

where the factor of $4 \pi$ arises from our normalization of the $S$-wave projected two-body $t$ matrix. The free propagator for

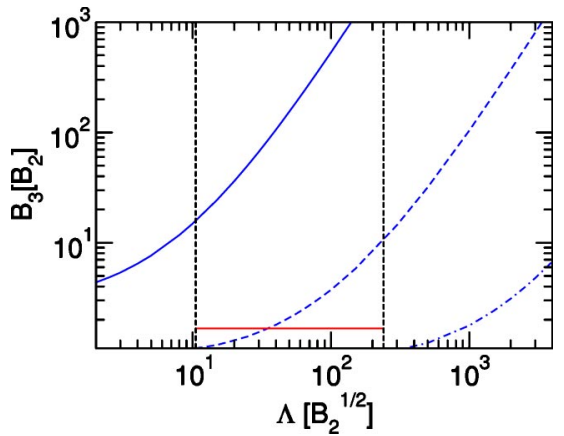

FIG. 1. The shallowest three-body binding energies indicated by the solid, dashed, and dash-dotted lines as a function of the momentum cutoff $\Lambda$. The vertical dashed line indicates the cutoff range in which the three-body system has exactly two bound states. The horizontal solid line shows the energy at which the shallowest three-body state is fixed.

three particles in their center of mass is given by

$$
G_{0}\left(u_{1}, u_{2}\right)=\left[E-E_{k i n}\right]^{-1}=\left[E-u_{1}^{2}-\frac{3}{4} u_{2}^{2}\right]^{-1},
$$

where $E_{\text {kin }}$ denotes the kinetic energy. Furthermore, it should be noted that the two-body propagator $\tau$ in Eq. (17) is evaluated at the energy in the corresponding two-body subsystem: $E-\frac{3}{4} u_{2}^{\prime 2}$. The permutation operator $P$ can be written as

$$
\begin{gathered}
\left\langle u_{1} u_{2}|P| u_{1}^{\prime} u_{2}^{\prime}\right\rangle=\int_{-1}^{1} d x \frac{\delta\left(u_{1}-\pi\left(u_{2}, u_{2}^{\prime}\right)\right) \delta\left(u_{1}^{\prime}-\pi\left(u_{2}^{\prime}, u_{2}\right)\right)}{\pi\left(u_{2}, u_{2}^{\prime}\right)^{2} \pi\left(u_{2}^{\prime}, u_{2}\right)^{2}} \\
\pi\left(u_{2}, u_{2}^{\prime}\right)=\sqrt{\frac{1}{4} u_{2}^{2}+u_{2}^{\prime 2}+u_{2} u_{2}^{\prime} x}
\end{gathered}
$$

Using this representation of $P$, we can write the integral equation as

$$
\begin{aligned}
\psi\left(u_{1}, u_{2}\right)= & 4 \pi G_{0}\left(u_{1}, u_{2}\right) g\left(u_{1}\right) \tau\left(E-\frac{3}{4} u_{2}^{2}\right) \\
& \times \int_{0}^{\infty} d u_{2}^{\prime} u_{2}^{\prime 2} \int_{-1}^{1} d x g\left(\pi\left(u_{2}, u_{2}^{\prime}\right)\right) \\
& \times \psi\left(\pi\left(u_{2}^{\prime}, u_{2}\right), u_{2}^{\prime}\right) .
\end{aligned}
$$

This is an homogeneous integral equation in two variables. It can be further simplified by defining a new function $F\left(u_{2}\right)$ of only one variable via

$$
\psi\left(u_{1}, u_{2}\right)=G_{0}\left(u_{1}, u_{2}\right) g\left(u_{1}\right) \tau\left(E-\frac{3}{4} u_{2}^{2}\right) F\left(u_{2}\right)
$$

leading to the integral equation 


$$
\begin{aligned}
F\left(u_{2}\right)= & 4 \pi \int_{0}^{\infty} d u_{2}^{\prime} u_{2}^{\prime 2} \int_{-1}^{1} d x g\left(\pi\left(u_{2}, u_{2}^{\prime}\right)\right) \\
& \times G_{0}\left(\pi\left(u_{2}^{\prime}, u_{2}\right), u_{2}^{\prime}\right) g\left(\pi\left(u_{2}^{\prime}, u_{2}\right)\right) \tau\left(E-\frac{3}{4} u_{2}^{\prime 2}\right) F\left(u_{2}^{\prime}\right) .
\end{aligned}
$$

The three-body binding energies are given by those values of $E$ for which Eq. (24) has a nontrivial solution. By expressing the two-body coupling constant $\lambda_{2}$ in terms of the binding energy of the shallow two-body bound state in Eq. (9), we have already renormalized the two-body problem.

The three-body system is stabilized against the Thomas collapse by the presence of the momentum cutoff $\Lambda$. No three-body force is required for this purpose. After the cutoff is introduced, there are no bound states with binding energies
$B_{3} \geq \Lambda^{2}{ }^{3}$ However, the three-body binding energies depend strongly on the value of the cutoff $\Lambda$. This is illustrated in Fig. 1 where the shallowest three-body binding energies are indicated by the solid, dashed, and dash-dotted lines. The three-body system has exactly two bound states in the cutoff range indicated by the two vertical dashed lines.

We now include the second term on the right-hand side of Eq. (14) which contains the three-body force. The derivation of an explicit representation in momentum space proceeds as for the first term. The full equation including both terms then reads

\footnotetext{
${ }^{3}$ This momentum cutoff $\Lambda$ can also be thought of as introducing a finite range $r \sim 1 / \Lambda$ for the pair potential in position space. The Thomas collapse is then stopped when the bound state size is of the same order as the range of the potential.
}

$$
\begin{aligned}
F\left(u_{2}\right)= & 4 \pi \int_{0}^{\infty} d u_{2}^{\prime} u_{2}^{\prime 2} \int_{-1}^{1} d x g\left(\pi\left(u_{2}, u_{2}^{\prime}\right)\right) G_{0}\left(\pi\left(u_{2}^{\prime}, u_{2}\right), u_{2}^{\prime}\right) g\left(\pi\left(u_{2}^{\prime}, u_{2}\right)\right) \tau\left(E-\frac{3}{4} u_{2}^{\prime 2}\right) F\left(u_{2}^{\prime}\right) \\
& +(4 \pi)^{3} \int_{0}^{\infty} d u_{1}^{\prime} u_{1}^{\prime 2} g\left(u_{1}^{\prime}\right) G_{0}\left(u_{1}^{\prime}, u_{2}\right) \tau_{3}(E) \xi\left(u_{1}^{\prime}, u_{2}\right)\left[\int_{0}^{\infty} d u_{1}^{\prime \prime} u_{1}^{\prime \prime 2} \int_{0}^{\infty} d u_{2}^{\prime \prime} u_{2}^{\prime \prime 2} \xi\left(u_{1}^{\prime \prime}, u_{2}^{\prime \prime}\right) g\left(u_{1}^{\prime \prime}\right) G_{0}\left(u_{1}^{\prime \prime}, u_{2}^{\prime \prime}\right) \tau\left(E-\frac{3}{4} u_{2}^{\prime \prime 2}\right) F\left(u_{2}^{\prime \prime}\right)\right. \\
& \left.+\int_{0}^{\infty} d u_{2}^{\prime \prime} u_{2}^{\prime \prime 2} \int_{0}^{\infty} d u_{2}^{\prime \prime \prime} u_{2}^{\prime \prime \prime 2} \int_{-1}^{1} d x \xi\left(\pi\left(u_{2}^{\prime \prime}, u_{2}^{\prime \prime \prime}\right), u_{2}^{\prime \prime}\right) g\left(\pi\left(u_{2}^{\prime \prime \prime}, u_{2}^{\prime \prime}\right)\right) G_{0}\left(\pi\left(u_{2}^{\prime \prime \prime}, u_{2}^{\prime \prime}\right), u_{2}^{\prime \prime \prime}\right) \tau\left(E-\frac{3}{4} u_{2}^{\prime \prime \prime 2}\right) F\left(u_{2}^{\prime \prime \prime}\right)\right]
\end{aligned}
$$

where $\xi\left(\mathbf{u}_{1}, \mathbf{u}_{2}\right) \equiv\left\langle\mathbf{u}_{1} \mathbf{u}_{2} \mid \xi\right\rangle$ is defined as

$$
\xi\left(\mathbf{u}_{1}, \mathbf{u}_{2}\right)=\exp \left(-\frac{u_{1}^{2}+\frac{3}{4} u_{2}^{2}}{\Lambda^{2}}\right) .
$$

Note that the term in the exponent is the kinetic energy of the three-body system. Thus it is exactly the kinetic energy flowing through the three-body interaction which is limited by the cutoff parameter $\Lambda$. This choice of the cutoff function satisfies Bose symmetry explicitly. The factors of $4 \pi$ and $(4 \pi)^{3}$ arise from our normalization of the $S$-wave projection of $t$ and $t_{3}$.

The value of the three-body force is determined by the renormalization condition that the shallowest bound state energy is fixed as the cutoff is varied. This value is denoted by the horizontal solid line in Fig. 1. Depending on the value of the cutoff, the three-body force then must provide additional attraction or repulsion in order to keep the shallowest boundstate energy fixed as the cutoff is varied. Thus one threebody datum (in our case the shallowest bound state energy) is required as input while all other low-energy three-body observables can be predicted. Once the shallowest bound state is fixed, the binding energies of the deeper bound states will also be cutoff independent. When the cutoff is increased and a new bound state appears at threshold, the three-body force must turn from strongly repulsive to strongly attractive to satisfy the renormalization condition for the shallowest bound state. The additional state is then added as a deep state rather than at threshold. Low-energy three-body observables are not affected by the additional deep bound states. As a consequence, the cutoff can be made arbitrarily large in the three-body system.

The renormalization procedure determines three-body coupling constant $\lambda_{3}\left(B_{3}, \Lambda\right)$ uniquely. It was used in Refs. $[7,8]$ to renormalize the three-body equation derived from nonrelativistic effective-field theory with an auxiliary field for the interacting two-particle state. See Ref. [28] for an earlier discussion of this renormalization method. While the part of Eq. (24) resulting from two-body interactions only is very similar to the corresponding part of the field-theoretical equation, the part containing the three-body force (in form of $\tau_{3}$ ) is more complicated. The simplicity of the fieldtheoretical equation is due the specific form of the threebody interaction using an auxiliary field in Refs. [7,8]. ${ }^{4}$

We now explicitly verify the renormalization of the threebody system as described above. By varying the cutoff parameter $\Lambda$ and tuning the three-body coupling $\lambda_{3}$ such that

\footnotetext{
${ }^{4}$ One would expect that using auxiliary fields would also simplify the four-body equations. However, it is not obvious how to treat the $(2)+(2)$ clusters in the intermediate state without introducing uncontrolled approximations.
} 


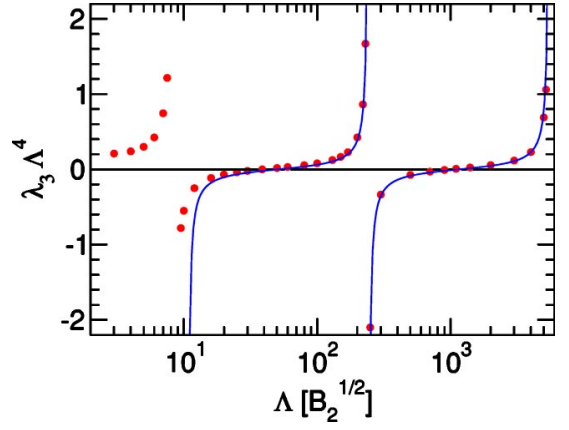

FIG. 2. The three-body coupling constant $\lambda_{3}$ as a function of the cutoff parameter $\Lambda$. The solid blue line shows a fit of Eq. (26) with $c=0.076$ and $L_{3}=23.3 \sqrt{B_{2}}$ to the points for $\Lambda \geqslant 245 \sqrt{B_{2}}$.

the shallowest three-body binding energy stays constant, we can determine the renormalization-group evolution of $\lambda_{3}$ numerically. For the dimensionless coupling constant $\lambda_{3} \Lambda^{4}$, we confirm the results of Refs. [7,8,12,13]: $\Lambda^{4} \lambda_{3}(\Lambda)$ shows a limit cycle behavior and is single valued. ${ }^{5}$ This limit cycle is shown in Fig. 2. For large values of the cutoff $\Lambda$, the dimensionless three-body coupling constant $\Lambda^{4} \lambda_{3}$ flows towards an ultraviolet limit cycle. For $\Lambda \rightarrow \infty$, it has the limiting behavior

$$
\lambda_{3}(\Lambda)=\frac{c}{\Lambda^{4}} \frac{\sin \left[s_{0} \ln \left(\Lambda / L_{3}\right)-\arctan \left(1 / s_{0}\right)\right]}{\sin \left[s_{0} \ln \left(\Lambda / L_{3}\right)+\arctan \left(1 / s_{0}\right)\right]},
$$

where $s_{0} \approx 1.00624$ is a transcendental number that determines the period of the limit cycle. If the cutoff $\Lambda$ is multiplied by a factor $\exp \left(n \pi / s_{0}\right) \approx(22.7)^{n}$ with $n$ an integer, the three-body coupling $\lambda_{3}$ is unchanged. $L_{3}$ is a three-body parameter generated by dimensional transmutation. One can either specify the dimensionless coupling constant $\Lambda^{4} \lambda_{3}(\Lambda)$ and the cutoff $\Lambda$ or the dimensionful three-body parameter $L_{3}$. The constant $c$ is universal and independent of $L_{3}$ within our numerical accuracy. We have determined $c$ numerically by fitting $\Lambda^{4} \lambda_{3}(\Lambda)$ for different three-body parameters $L_{3}$ to Eq. (26) and found

$$
c=0.074 \pm 0.003,
$$

where the error has been estimated from the observed variation in the fit results for $c$. The three-body parameter $L_{3}$ can be determined by fixing a three-body binding energy $B_{3}$. Of course, one could also use a three-body binding energy directly to characterize the value of the three-body coupling $\lambda_{3}$ at a given cutoff. However, it is advantageous to use $L_{3}$ because Eq. (26) takes a particularly simple form in terms of $L_{3}$.

One might expect that the smooth Gaussian regulator function we use would lead to better behaved numerical solutions of the three-body equations than the sharp cutoff used for the field-theoretical equation in Refs. [7,8]. However, it turns out that the convergence of the three-body coupling to the ultraviolet limit cycle is significantly slower than in the

\footnotetext{
${ }^{5}$ Note that $\lambda_{3}$ is defined with the opposite sign of the three-body coupling constant $H$ in the field theory formulation [7,8].
}

effective-field theory formulation. This is due to the more complicated structure of the part of Eq. (24) containing the three-body force. For very large loop momenta, the threebody part of Eq. (24) simplifies considerably and has a similar form as in the EFT formulation. In this limit, it is possible to derive the general form of Eq. (26).

In general, we recover the results for the three-body binding energies from Refs. [7,8,12,13]. However, we note that somewhat higher cutoffs are required to reach convergent results for the deeper three-body bound states. The numerical effort for the solution of the three-body equations becomes larger as the value of the three-body coupling $\lambda_{3}$ is increased. This is also related to the relatively complicated structure of the part of Eq. (24) containing the three-body force.

\section{The four-body sector}

We now turn to the four-body sector. The four-body binding energies are given by the nontrivial solutions of the Yakubovsky equations [25] which are based on a generalization of the decomposition into Faddeev components for the three-body system. The full four-body wave function $\Psi$ is first decomposed into Faddeev components, followed by a second decomposition into so-called "Yakubovsky components." In the case of identical bosons, one ends up with two Yakubovsky components $\psi_{A}$ and $\psi_{B}$. We start from the Yakubovsky equations including a general three-body force in the form written down by Glöckle and Kamada [41]. The full four-body bound-state wave function is decomposed into the Yakubovsky components $\psi_{A}$ and $\psi_{B}$ via

$$
\Psi=\left[1+(1+P) P_{34}\right](1+P) \psi_{A}+(1+P)(1+\widetilde{P}) \psi_{B},
$$

where $P_{i j}$ exchanges particles $i$ and $j, P$ is defined in Eq. (10), and $\widetilde{P}$ is given by

$$
\widetilde{P}=P_{13} P_{24}
$$

The equations for the two wave function components read

$$
\begin{gathered}
\psi_{A}=G_{0} t_{12} P\left[\left(1+P_{34}\right) \psi_{A}+\psi_{B}\right]+\frac{1}{3}\left(1+G_{0} t_{12}\right) G_{0} V_{3} \Psi, \\
\psi_{B}=G_{0} t_{12} \tilde{P}\left[\left(1+P_{34}\right) \psi_{A}+\psi_{B}\right],
\end{gathered}
$$

where $t_{12}$ denotes the two-body $t$ matrix for particles 1 and 2 and $V_{3}$ is the three-body force defined in Eq. (12). Note that the three-body force couples to the full four-body wave function $\Psi$. The factor of one-third in front of the three-body force term arises because we insert the full three-body interaction for $V_{3}$. This is possible since we consider three-body contact interactions which are symmetric under the exchange of any pair of particles.

In order to describe the four-body system at rest, three Jacobi momenta are required. The structure of the four-body equations is more complex than that of the three-body equations because both $(3)+(1)$ and $(2)+(2)$ fragmentations can 
occur. As a consequence, two different sets of Jacobi momenta are required. The (3)+(1) fragmentation is described by the vector

$$
\mathbf{u}_{\mathbf{3}}=\frac{3}{4}\left(\mathbf{k}_{\mathbf{4}}-\frac{1}{3}\left(\mathbf{k}_{\mathbf{1}}+\mathbf{k}_{\mathbf{2}}+\mathbf{k}_{\mathbf{3}}\right)\right)
$$

along with the Jacobi momenta for the three-body system given in Eq. (15). The (2)+(2) fragmentation is described by the set

$$
\begin{gathered}
\mathbf{v}_{\mathbf{1}}=\frac{1}{2}\left(\mathbf{k}_{\mathbf{1}}-\mathbf{k}_{\mathbf{2}}\right), \quad \mathbf{v}_{\mathbf{2}}=\frac{1}{2}\left(\mathbf{k}_{\mathbf{1}}+\mathbf{k}_{\mathbf{2}}\right)-\frac{1}{2}\left(\mathbf{k}_{\mathbf{3}}+\mathbf{k}_{\mathbf{4}}\right), \\
\text { and } \quad \mathbf{v}_{\mathbf{3}}=\frac{1}{2}\left(\mathbf{k}_{\mathbf{3}}-\mathbf{k}_{\mathbf{4}}\right) .
\end{gathered}
$$

Depending on which Yakubovsky component and operator in Eq. (30) is concerned, one of the two sets of coordinates will be more convenient than the other.

As in the previous subsection, we will only display the derivation of the analytic expression for the Yakubovsky equations without the three-body force. It is natural to evaluate the Yakubovsky component $\psi_{A}$ as a function of $u_{1}, u_{2}$, and $u_{3}$ :

$$
\begin{aligned}
\left\langle u_{1} u_{2} u_{3} \mid \psi_{A}\right\rangle= & 4 \pi G_{0}\left(u_{1}, u_{2}, u_{3}\right) g\left(u_{1}\right) \tau\left(E-\frac{3}{4} u_{2}^{2}-\frac{2}{3} u_{3}^{2}\right)\left[\int d u_{2}^{\prime} u_{2}^{\prime 2} \int_{-1}^{1} d x g\left(\pi\left(u_{2}, u_{2}^{\prime}\right)\right)\left\langle\pi\left(u_{2}^{\prime}, u_{2}\right) u_{2}^{\prime} u_{3} \mid \psi_{A}\right\rangle\right. \\
& +\frac{1}{2} \int d u_{2}^{\prime} u_{2}^{\prime 2} \int_{-1}^{1} d x \int_{-1}^{1} d x^{\prime} g\left(\pi\left(u_{2}, u_{2}^{\prime}\right)\right)\left\langle\pi\left(u_{2}^{\prime}, u_{2}\right) \widetilde{u}_{2}\left(u_{2}^{\prime}, u_{3}\right) \widetilde{u}_{3}\left(u_{2}^{\prime}, u_{3}\right) \mid \psi_{A}\right\rangle \\
& \left.+\frac{1}{2} \int d u_{2}^{\prime} u_{2}^{\prime 2} \int_{-1}^{1} d x \int_{-1}^{1} d x^{\prime} g\left(\pi\left(u_{2}, u_{2}^{\prime}\right)\right) \times\left\langle\pi\left(u_{2}^{\prime}, u_{2}\right) \widetilde{v}_{2}\left(u_{2}^{\prime}, u_{3}\right) \widetilde{v}_{3}\left(u_{2}^{\prime}, u_{3}\right) \mid \psi_{B}\right\rangle\right],
\end{aligned}
$$

where the two-body propagator $\gamma\left(E-\frac{3}{4} u_{2}^{2}-\frac{2}{3} u_{3}^{2}\right)$ is again evaluated at the energy of the corresponding two-body subsystem. $G_{0}\left(u_{1}, u_{2}, u_{3}\right)$ is the free four-particle propagator in the center-of-mass system of the four particles and $\pi\left(u_{2}, u_{2}^{\prime}\right)$ is defined in Eq. (20). The second Yakubovsky component $\psi_{B}$ is computed as a function of the momenta $v_{1}, v_{2}$, and $v_{3}$ :

$$
\begin{aligned}
\left\langle v_{1} v_{2} v_{3} \mid \psi_{B}\right\rangle= & 4 \pi G_{0}\left(v_{1}, v_{2}, v_{3}\right) g\left(v_{1}\right) \tau\left(E-\frac{1}{2} v_{2}^{2}-v_{3}^{3}\right) \\
& \times\left[\int d v_{3}^{\prime} v_{3}^{\prime 2} g\left(v_{3}^{\prime}\right)\left\langle v_{3} v_{2} v_{3}^{\prime} \mid \psi_{B}\right\rangle\right. \\
& +\int d v_{3}^{\prime} v_{3}^{\prime 2} \int_{-1}^{1} d x g\left(v_{3}^{\prime}\right)\left\langle v_{3} \bar{u}_{2}\left(v_{2}, v_{3}^{\prime}\right) \bar{u}_{3}\left(v_{2}, v_{3}^{\prime}\right)\right. \\
& \left.\times\left|\psi_{A}\right\rangle\right] .
\end{aligned}
$$

In Eqs. (33) and (34), we have used the abbreviations

$$
\begin{gathered}
\tilde{u}_{2}\left(u_{2}, u_{3}\right)=\sqrt{\frac{1}{9} u_{2}^{2}+\frac{64}{81} u_{3}^{2}+\frac{16}{27} u_{2} u_{3} x^{\prime}} \\
\tilde{u}_{3}\left(u_{2}, u_{3}\right)=\sqrt{u_{2}^{2}+\frac{1}{9} u_{3}^{2}-\frac{2}{3} u_{2} u_{3} x^{\prime}} \\
\tilde{v}_{2}\left(u_{2}, u_{3}\right)=\sqrt{u_{2}^{2}+\frac{4}{9} u_{3}^{2}+\frac{4}{3} u_{2} u_{3} x^{\prime}},
\end{gathered}
$$

$$
\begin{aligned}
& \widetilde{v}_{3}\left(u_{2}, u_{3}\right)=\sqrt{\frac{1}{4} u_{2}^{2}+\frac{4}{9} u_{3}^{2}-\frac{2}{3} u_{2} u_{3} x^{\prime},} \\
& \bar{u}_{2}\left(v_{2}, v_{3}\right)=\sqrt{\frac{4}{9} v_{2}^{2}+\frac{4}{9} v_{3}^{2}-\frac{8}{9} v_{2} v_{3} x^{\prime}}, \\
& \bar{u}_{3}\left(v_{2}, v_{3}\right)=\sqrt{\frac{1}{4} v_{2}^{2}+v_{3}^{2}+v_{2} v_{3} x^{\prime}} .
\end{aligned}
$$

Similar to the three-body case, Eqs. (33) and (34) can be simplified from two coupled integral equations in three variables to two coupled integral equations in two variables. Performing the substitutions

$$
\begin{aligned}
& \left\langle u_{1} u_{2} u_{3} \mid \psi_{A}\right\rangle=g\left(u_{1}\right) G_{0}\left(u_{1}, u_{2}, u_{3}\right) F_{A}\left(u_{2}, u_{3}\right), \\
& \left\langle v_{1} v_{2} v_{3} \mid \psi_{B}\right\rangle=g\left(v_{1}\right) G_{0}\left(v_{1}, v_{2}, v_{3}\right) F_{B}\left(v_{2}, v_{3}\right),
\end{aligned}
$$

we obtain the integral equations for $F_{A}\left(u_{2}, u_{3}\right)$ and $F_{B}\left(v_{2}, v_{3}\right)$ : 


$$
\begin{aligned}
F_{A}\left(u_{2}, u_{3}\right)= & 4 \pi \tau\left(E-\frac{3}{4} u_{2}^{2}-\frac{2}{3} u_{3}^{2}\right)\left[\int d u_{2}^{\prime} u_{2}^{\prime 2} \int_{-1}^{1} d x g\left(\pi\left(u_{2}, u_{2}^{\prime}\right)\right) g\left(\pi\left(u_{2}^{\prime}, u_{2}\right)\right)\right. \\
& \times G_{0}\left(\pi\left(u_{2}^{\prime}, u_{2}\right), u_{2}^{\prime}, u_{3}\right) F_{A}\left(u_{2}^{\prime}, u_{3}\right)+\frac{1}{2} \int d u_{2}^{\prime} u_{2}^{\prime 2} \int_{-1}^{1} d x \int_{-1}^{1} d x^{\prime} g\left(\pi\left(u_{2}, u_{2}^{\prime}\right)\right) g\left(\pi\left(u_{2}^{\prime}, u_{2}\right)\right) \\
& \times G_{0}\left(\pi\left(u_{2}^{\prime}, u_{2}\right), \tilde{u}_{2}\left(u_{2}^{\prime}, u_{3}\right), \tilde{u}_{3}\left(u_{2}^{\prime}, u_{3}\right)\right) F_{A}\left(\tilde{u}_{2}\left(u_{2}^{\prime}, u_{3}\right), \tilde{u}_{3}\left(u_{2}^{\prime}, u_{3}\right)\right) \\
& +\frac{1}{2} \int d u_{2}^{\prime} u_{2}^{\prime 2} \int_{-1}^{1} d x \int_{-1}^{1} d x^{\prime} g\left(\pi\left(u_{2}, u_{2}^{\prime}\right)\right) g\left(\pi\left(u_{2}^{\prime}, u_{2}\right)\right) \\
& \left.\times G_{0}\left(\pi\left(u_{2}^{\prime}, u_{2}\right), \widetilde{v}_{2}\left(u_{2}^{\prime}, u_{3}\right), \widetilde{v}_{3}\left(u_{2}^{\prime}, u_{3}\right)\right) F_{B}\left(\widetilde{v}_{2}\left(u_{2}^{\prime}, u_{3}\right), \widetilde{v}_{3}\left(u_{2}^{\prime}, u_{3}\right)\right)\right], \\
F_{B}\left(v_{2}, v_{3}\right)= & 4 \pi \tau\left(E-\frac{1}{2} v_{2}^{2}-v_{3}^{2}\right)\left[\int d v_{3}^{\prime} v_{3}^{\prime 2} g\left(v_{3}^{\prime}\right) g\left(v_{3}\right) G_{0}\left(v_{3}, v_{2}, v_{3}^{\prime}\right) F_{B}\left(v_{2}, v_{3}^{\prime}\right)\right. \\
& +\int d v_{3}^{\prime} v_{3}^{\prime 2} \int_{-1}^{1} d x g\left(v_{3}^{\prime}\right) g\left(v_{3}\right) G_{0}\left(v_{3}, \bar{u}_{2}\left(v_{2}, v_{3}^{\prime}\right), \bar{u}_{3}\left(v_{2}, v_{3}^{\prime}\right)\right) \\
& \left.\times F_{A}\left(\bar{u}_{2}\left(v_{2}, v_{3}^{\prime}\right), \bar{u}_{3}\left(v_{2}, v_{3}^{\prime}\right)\right)\right] .
\end{aligned}
$$

The inclusion of the three-body force term is straightforward but lengthy and the corresponding expressions are given in the Appendix.

In order to obtain the four-body binding energies, we have to solve the Yakubovsky equations with the three-body force term. The binding energies can be found by discretizing the above equations and calculating the eigenvalues of the resulting matrix. They are given by the energies at which any eigenvalue of the matrix is equal to 1 . The wave function is then given by the corresponding eigenvector.

The renormalization analysis of the four-body system is complicated by the cutoff dependence of the number of bound states in the three-body subsystems. The further the cutoff $\Lambda$ is increased, the more three-body bound states appear. While the spurious deep three-body states have no influence on low-energy three-body observables, they create an instability in the four-body system which can collapse into a deep three-body bound state plus another particle. This limits cutoff variations to an interval $\Lambda_{0}<\Lambda<22.7 \Lambda_{0}$ for some $\Lambda_{0}$, in which the number of three-body bound states remains constant. Since the cutoff can still be varied by more than a factor of 10, we are nevertheless able to study the renormalization properties and obtain converged numerical results. Alternatively, one could explicitly subtract out the spurious bound states from the three-body $t$ matrix. We will come back to this question in the next section.

\section{RENORMALIZATION AND NUMERICAL RESULTS}

In this section, we will discuss the renormalization of the four-boson system and present some numerical results for the four-body system of ${ }^{4} \mathrm{He}$ atoms. For convenience, we will set
Boltzmann's constant to unity: $k=1$. Since the scattering length of ${ }^{4} \mathrm{He}$ atoms is much larger than their effective range, they are an ideal application for our theory and a leadingorder calculation should be accurate to about $10 \%$ (since $l / a \simeq 10 \%$ ).

The quantitative experimental information on low-energy ${ }^{4} \mathrm{He}$ atoms, however, is rather limited. Using diffraction of a molecular beam of small ${ }^{4} \mathrm{He}$ clusters from a transmission grating, the bond length of the ${ }^{4} \mathrm{He}$ dimer has been measured to be $\langle r\rangle=(52 \pm 4) \AA$ [42]. This value is an order magnitude larger than their effective range $r_{e} \approx 7 \AA$, which can be taken as an estimate of the natural low-energy length scale $l$. The scattering length $a=\left(104_{-18}^{+8}\right) \AA$ and the dimer binding energy $B_{2}=\left(1.1_{-0.2}^{+0.3}\right) \mathrm{mK}$ were derived from the measured bond length using the zero range approximation [42]. The ${ }^{4} \mathrm{He}$ trimer, tetramer, and several larger ${ }^{4} \mathrm{He}$ clusters have been observed $[43,44]$, but no quantitative experimental information about their binding energies is available to date.

However, there is a large number of theoretical calculations using realistic ${ }^{4} \mathrm{He}$ potentials for the trimer $\left({ }^{4} \mathrm{He}_{3}\right)$. These calculations typically predict a trimer ground state with an energy of about $120 \mathrm{mK}$ and one excited state with a binding energy of about $2 \mathrm{mK}$ [45-48]. The ground and excited states of the tetramer $\left({ }^{4} \mathrm{He}_{4}\right)$ and larger clusters have been calculated by Blume and Greene (BG) [21]. They have used the LM2M2 potential [49] and a combination of Monte Carlo methods and the adiabatic hyperspherical approximation. Their results for the trimer energies agree with the exact three-body calculations of Refs. [45-48].

In the absence of quantitative experimental information on the three-body clusters, we take the binding energy of the ${ }^{4} \mathrm{He}$ trimer excited state from theoretical calculations using 


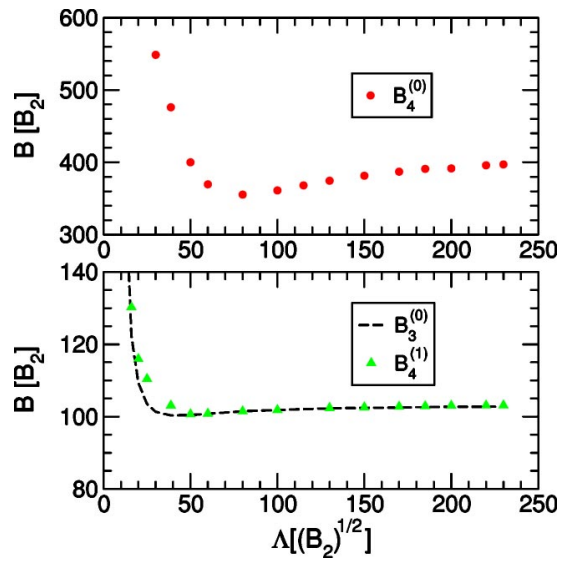

FIG. 3. Binding energies of the three- and four-body system as a function of the cutoff $\Lambda . B_{n}^{(0)}$ and $B_{n}^{(1)}$ denote the ground and first excited state of the $n$-body system.

the LM2M2 potential as input to fix $L_{3}$. We use the value of Blume and Greene: $B_{3}^{(1)} / B_{2}=1.767$ for this purpose [20,21]. We can then calculate the trimer ground state and the tetramer binding energies for the LM2M2 potential based on low-energy universality. Before we present our numerical results for the ${ }^{4} \mathrm{He}_{4}$ system, we discuss the renormalization of the four-body problem.

In Fig. 3, we have plotted the three-body ground state energy $B_{3}^{(0)}$ and the four-body energies as a function of the cutoff $\Lambda$. As in the case of the trimer, the ${ }^{4} \mathrm{He}$ tetramer has a ground state $B_{4}^{(0)}$ and one excited state $B_{4}^{(1)}$. The cutoff dependence of $B_{3}^{(0)}$ must flatten out and reach a plateau as $\Lambda$ is increased since the three-body system was renormalized by fixing the trimer excited-state binding energy $B_{3}^{(1)}$ (cf. Sec. II B). However, Fig. 3 shows that the four-body binding energies $B_{4}^{(0)}$ and $B_{4}^{(1)}$ also reach a plateau as $\Lambda$ is increased. The excited-state energy $B_{4}^{(1)}$ has a negligible cutoff dependence already at fairly small cutoffs. For the ground state $B_{4}^{(0)}$, the situation is somewhat more complicated and the cutoff dependence of $B_{4}^{(0)}$ reaches a plateau only at the largest cutoff values calculated. The residual cutoff dependence is about $2 \%$ for the excited state and $5 \%$ for the ground state. A slower convergence for the ground state is expected since the value for $B_{4}^{(0)}$ is a factor four larger than for the excited state and finite cutoff effects of the order $\sqrt{|E|} / \Lambda$ are more important. The residual cutoff dependence for both states is at least a factor 2 smaller than the corrections from higher orders in the expansion in $l / a$ which are expected to be of the order $l / a \sim 10 \%$. We speculate that higher precision could be achieved by increasing the cutoff further. As already noted earlier, this would create unphysical three-body bound states which create an instability in the four-body system. These states would have to be subtracted explicitly. While such a subtraction is possible, this is beyond the scope of our paper and we will not attempt such a subtraction here.

Taken together, the above observations provide strong numerical evidence that the four-body binding energies are cutoff independent up to higher-order corrections in $l / a$. In particular, a four-body force with limit cycle behavior would lead to a much stronger cutoff dependence of the binding
TABLE I. Binding energies of the ${ }^{4} \mathrm{He}$ trimer and tetramer in $\mathrm{mK}$. The two right columns show the results by Blume and Greene [21] (denoted by the index BG) while the two left columns show our results. The number in brackets was used as input to fix $L_{3}$.

\begin{tabular}{lcccc}
\hline \hline System & $B^{(0)}(\mathrm{mK})$ & $B^{(1)}(\mathrm{mK})$ & $B_{\mathrm{BG}}^{(0)}(\mathrm{mK})$ & $B_{\mathrm{BG}}^{(1)}(\mathrm{mK})$ \\
\hline${ }^{4} \mathrm{He}_{3}$ & 127 & {$[2.186]$} & 125.5 & 2.186 \\
${ }^{4} \mathrm{He}_{4}$ & 492 & 128 & 559.7 & 132.7 \\
\hline \hline
\end{tabular}

energies (cf. Fig. 1) and can be excluded. The occurrence of the plateaus for $B_{4}^{(0)}$ and $B_{4}^{(1)}$ in Fig. 3 suggests that a fourbody force is not required for renormalization of the fourbody system at leading order in $l / a$. Renormalization of the three-body system automatically generates cutoffindependent results for the four-body binding energies. As a consequence, the four-body binding energies can be predicted from two- and three-body input alone.

We now turn to our numerical results for the four-body system of ${ }^{4} \mathrm{He}$ atoms. From the plateaus in Fig. 3, we can read off the values of the binding energies. A comparison of our results with the values obtained by BG [21] is shown in Table I. The results of their calculation for the trimer and tetramer are given in the two right columns of Table I, while our results are given in the two left columns. In general, our results are in good agreement with the values of BG. For the trimer ground-state and the tetramer excited-state energies, we obtain the values $B_{3}^{(0)}=127 \mathrm{mK}$ and $B_{4}^{(1)}=128 \mathrm{mK}$, respectively. For the tetramer ground state, we obtain $B_{4}^{(0)}$ $=492 \mathrm{mK}$. While the value of $B_{4}^{(0)}$ is already relatively large, it is still a factor 3 smaller than the natural four-body energy scale $\sim 1.5 \mathrm{~K}$ where the effective theory description is expected to break down.

The natural energy scales can be estimated as follows: For two particles, it is directly determined by the natural length scale $l$ and the mass $M$ of the particles: $e_{2} \sim \hbar^{2} /\left(M l^{2}\right)$ $\approx 250 \mathrm{mK}$. For three and four particles, this estimate should be scaled according to the number of pairs available, ${ }^{6}$ leading to the values $e_{3} \sim 750 \mathrm{mK}$ and $e_{4} \sim 1.5 \mathrm{~K}$. This estimate can be made for cutoff values at which the three-body force vanishes. Since all observables are independent of the cutoff, however, it is valid for arbitrary cutoffs. Our short-range effective theory can describe $n$-body bound states with binding energies $B_{n} \ll e_{n}$. For deeper bound states closer to the natural energy scale the errors are expected to increase.

The values in Table I have been computed at a cutoff of $\Lambda=235 \sqrt{B_{2}}$ which is close to the largest possible value with only two three-body bound states. Our values for $B_{4}^{(0)}$ and $B_{4}^{(1)}$ agree with the $\mathrm{BG}$ values to within $12 \%$ and $3 \%$, respectively. The dominant correction to our results is due to effective range effects which are not included in our leading-order calculation. These deviations are within the expected accuracy of the effective theory. We expect the effective range corrections to the leading-order result to be of the order $r_{e} / a \sim 10 \%$. From the residual cutoff dependence, we esti-

\footnotetext{
${ }^{6} \mathrm{We}$ are grateful to Eric Braaten for suggesting to us this scaling of the natural energy scale according to the number of pairs.
} 
mate the numerical error of our calculation to be of the order of $2 \%$ for the excited state and $5 \%$ for the ground state. For fixed value of the cutoff $\Lambda$, our calculations are numerically accurate to three significant digits.

The large scattering length of ${ }^{4} \mathrm{He}$ atoms also leads to universal properties in the four-body problem. A typical example is the existence of correlations between different observables. These correlations become manifest in universal scaling functions relating dimensionless combinations of observables. Various scaling functions for the three-body system of ${ }^{4} \mathrm{He}$ atoms were studied in Refs. [26,50,51]. Here, we consider the four-body binding energies as a function of the three-body binding energies. In phenomenological calculations of the four-nucleon system, an approximately linear correlation between the three- and four-nucleon binding energies for various nucleon-nucleon potentials is observed: the Tjon line [14]. This correlation is approximately linear for the relevant range of binding energies [30]. Since the ${ }^{4} \mathrm{He}$ trimer and tetramer have both a ground state and an excited state, there are four "Tjon lines" in this case.

In Fig. 4, we show the correlations between the groundand excited-state energies of the ${ }^{4} \mathrm{He}$ trimer and tetramer. The first and third figure from the top show the energies of the tetramer excited state $B_{4}^{(1)}$ and ground state $B_{4}^{(0)}$ as a function of trimer excited-state energy $B_{3}^{(1)}$, respectively, while the second and fourth figure from the top show the same quantities as a function of the trimer ground-state energy $B_{3}^{(0)}$. The solid line is the leading-order result of our effective theory calculation and the cross denotes the result of the calculation by Blume and Greene for the LM2M2 potential [21]. For the ground states of the trimer and tetramer, calculations with other ${ }^{4} \mathrm{He}$ potentials are available as well. As an example, we show the results for the TTY, HFD-B, and HFDHE2 potentials taken from Refs. [17,20].

Similar to the nuclear sector, we find an approximately linear correlation over the range of binding energies relevant to ${ }^{4} \mathrm{He}$ atoms. The calculations for the realistic ${ }^{4} \mathrm{He}$ potentials fall close to the universal scaling curves from our effective theory. For the correlation between the ground-state energies, the Tjon line is directly evident in the potential model calculations shown in Fig. 4. If calculations with other potentials were performed for the excited-state energies, they would also fall on a line parallel to the universal scaling curve from effective theory. The deviation of calculations using realistic potentials from the universal line is mainly governed by effective range corrections which are expected to be of the order $10 \%$ for ${ }^{4} \mathrm{He}$ atoms. For the tetramer ground state, this deviation is about a factor 4 larger than for the excited state.

While the correlations in Fig. 4 are approximately linear over the range of binding energies calculated, we expect them to become nonlinear for a sufficiently large range of binding energies. Similar nonlinearities were observed for correlations between three-body observables in Refs. $[8,26]$. Our results suggest that the Tjon lines are universal properties of few-body systems with short-range interactions and large scattering length. They do not depend on the details of the short-distance physics which are very different in atomic and nuclear systems. It is interesting to note that in nuclear physics only a correlation between the ground-state energies
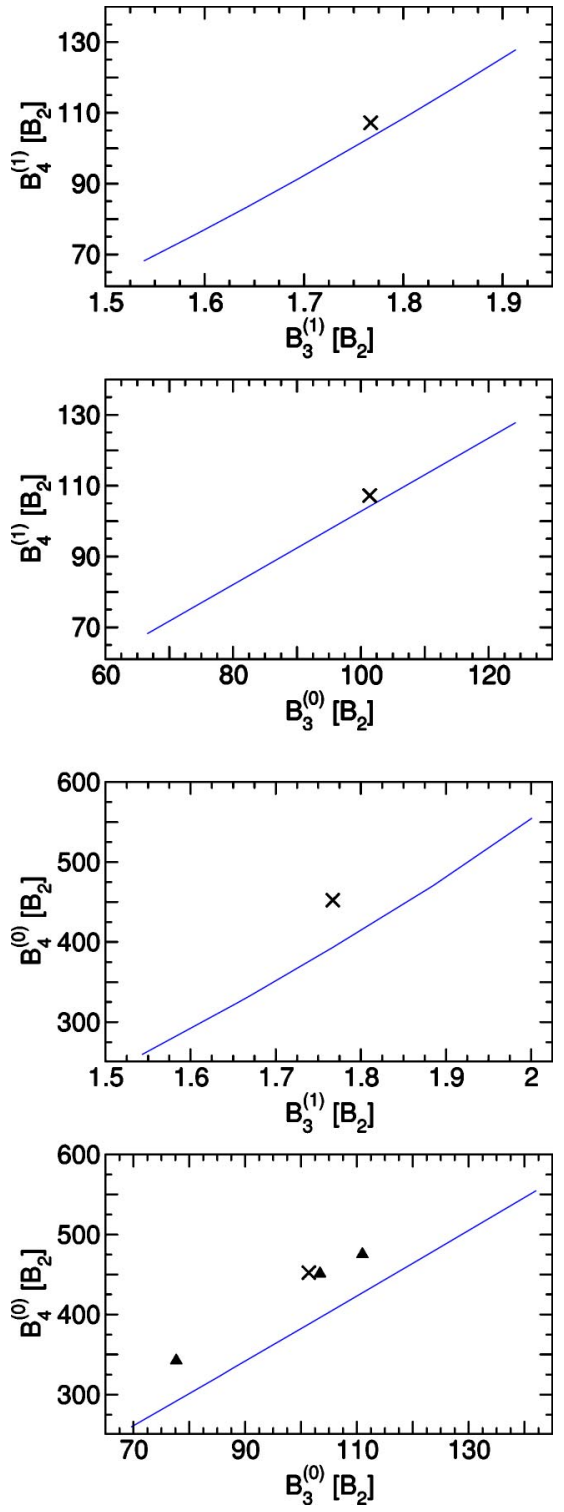

FIG. 4. The correlations between the ground- and excited-state energies of the ${ }^{4} \mathrm{He}$ trimer and tetramer. First and third figure from the top: the four-body excited-state energy $B_{4}^{(1)}$ and ground-state energy $B_{4}^{(0)}$ as a function of the three-body excited-state energy $B_{3}^{(1)}$. The second and fourth figure from the top: the same quantities as a function of three-body ground-state energy $B_{3}^{(0)}$. The solid line shows the leading-order effective theory result and the cross denotes the calculation for the LM2M2 potential by Blume and Greene [21]. The triangles show the results for the TTY, HFD-B, and HFDHE2 potentials [17,20].

of the three- and four-body systems has been observed. We expect this correlation to hold for all $S$-wave states that are within the range of validity of an effective theory with contact interactions. It would be interesting to see whether such a correlation also holds for the excited $J^{P}=0^{+}$state of the $\alpha$ particle above the $p+{ }^{3} \mathrm{H}$ threshold.

We have fitted the scaling functions shown in Fig. 4 with linear expressions and obtained 


$$
\begin{gathered}
\frac{B_{4}^{(0)}}{B_{2}}=-24.752+4.075 \frac{B_{3}^{(0)}}{B_{2}}, \quad 69 \leqslant \frac{B_{3}^{(0)}}{B_{2}} \leqslant 142, \\
\frac{B_{4}^{(0)}}{B_{2}}=-742.0+645.1 \frac{B_{3}^{(1)}}{B_{2}}, \quad 1.54 \leqslant \frac{B_{3}^{(1)}}{B_{2}} \leqslant 2.00, \\
\frac{B_{4}^{(1)}}{B_{2}}=-0.662+1.034 \frac{B_{3}^{(0)}}{B_{2}}, \quad 65 \leqslant \frac{B_{3}^{(0)}}{B_{2}} \leqslant 125, \\
\frac{B_{4}^{(1)}}{B_{2}}=-178.0+159.4 \frac{B_{3}^{(1)}}{B_{2}}, \quad 1.52 \leqslant \frac{B_{3}^{(1)}}{B_{2}} \leqslant 1.92 .
\end{gathered}
$$

These relations can be used to predict the tetramer groundand excited-state energies for any potential for which one of the trimer energies and the dimer binding energy are known. The expressions (39)-(42) are of the same accuracy as our explicit calculations (see the discussion above). They are expected to be most accurate for the excited states.

\section{SUMMARY AND OUTLOOK}

In this paper, we have studied the four-body system with short-range interactions and large scattering length. We have concentrated on the bound-state problem of four bosons starting from the Yakubovsky equations [25]. We have constructed an effective interaction potential including both a two- and three-body contact interaction. This is the minimal set of contact interactions required for renormalization of the three-body problem $[7,8]$. The two parameters of the effective potential were determined from matching to the binding energy of the dimer and the excited state of the trimer. We have then solved the four-body bound-state problem under the assumption that no four-body interaction is required for renormalization at leading order. We found that after renormalizing the two- and three-body subsystems, the four-body binding energies were automatically independent of the ultraviolet cutoff. This result suggests that the four-body interaction is not of leading order and the low-energy four-body observables are determined by properties of the two- and three-body systems up to corrections suppressed by $l /|a|$. Although we have considered only the four-boson bound state problem with large scattering length explicitly, we expect this result to hold for all low-energy four-body observables.

We have applied this effective theory to the four-body system of ${ }^{4} \mathrm{He}$ atoms and calculated the ground- and excitedstate energies of the ${ }^{4} \mathrm{He}$ tetramer. In the absence of experimental information on the ${ }^{4} \mathrm{He}$ trimer, we have taken the excited-state energy of the ${ }^{4} \mathrm{He}$ trimer as calculated by Blume and Greene for the LM2M2 potential [21] as input to determine the three-body parameter $L_{3}$. For the binding energies of the trimer ground state and the tetramer ground and excited states, we find the values $B_{3}^{(0)}=127 \mathrm{mK}, B_{4}^{(1)}$ $=128 \mathrm{mK}$, and $B_{4}^{(0)}=492 \mathrm{mK}$, respectively. The latter energy is still about a factor 3 smaller than the natural four-body energy scale $\sim 1.5 \mathrm{~K}$ where the effective theory is expected to break down. Our values for $B_{4}^{(0)}$ and $B_{4}^{(1)}$ agree with the calculation by Blume and Greene to within $12 \%$ and $3 \%$, respectively. These deviations are consistent with the expected accuracy at leading order in the large scattering length of about $l /|a| \sim 10 \%$.

The large scattering length of ${ }^{4} \mathrm{He}$ atoms leads to universal properties such as universal scaling functions. We have calculated the universal scaling functions relating the tetramer energies to the trimer energies. The correlations are approximately linear in the region of binding energies relevant for ${ }^{4} \mathrm{He}$ atoms. As expected from low-energy universality, the results of various calculations using realistic ${ }^{4} \mathrm{He}$ potentials fall close to the universal scaling curves. Corrections to the scaling curves are mainly governed by effective range effects. We have fitted the calculated scaling functions with linear expressions (39)-(42) that can be used to obtain the tetramer binding energies at leading order in $l /|a|$ for any potential if one of the trimer binding energies is known.

There are a number of directions that should be pursued in future work. While we have demonstrated that a four-body force is not necessary to renormalize the four-body system to leading order, the general power counting for four-body forces is still not understood. At which order does the leading four-body interaction enter? In the three-body system, e.g., the first-order correction is due to the two-body effective range. If a similar situation holds in the four-body system, it would be possible to predict low-energy four-body observables up to corrections of order $(l / a)^{2}$ from two- and threebody information alone.

The extension of the effective theory to calculate fourbody scattering observables would be very valuable. The knowledge of the dimer-dimer scattering length, for example, is important for experiments with ultracold atoms. For the simpler problem of fermions with two spin states (where the three-body parameter $L_{3}$ does not contribute), the dimer-dimer scattering length was recently calculated [52].

Whether this effective theory can be applied to the nuclear four-body system like the pionful theory [31] is an open question. While it is straightforward to generalize the effective theory to include spin and isospin, it is not clear whether an effective theory without explicit pions will be adequate for the $\alpha$ particle ground state with a binding energy of about $28 \mathrm{MeV}$. This question deserves further study. The effective theory might also help to shed some light on the renewed speculations about the existence of a shallow tetraneutron bound state [53].

\section{ACKNOWLEDGMENTS}

We thank E. Braaten, W. Glöckle, U. van Kolck, and A. Nogga for valuable discussions and E. Braaten and A. Nogga for comments on the manuscript. This research was supported in part by DOE Grant No. DE-FG02-00ER41132.

\section{APPENDIX: YAKUBOVSKY EQUATIONS WITH THREE-BODY FORCE}

In this appendix, we derive the analytic expressions for the three-body force term in the Yakubovsky equations (30) in momentum space. The three-body force term 


$$
\frac{1}{3}\left\langle u_{1} u_{2} u_{3}\left|\left(1+G_{0} t_{12}\right) G_{0} V_{3}\right| \Psi\right\rangle
$$

couples to the full four-body wave function $\Psi$ [cf. Eq. (28) ], which is related to the Yakuvosky components $\psi_{A}$ and $\psi_{B}$ via

$$
\begin{aligned}
\Psi= & \left(1+P_{34}+P P_{34}+P+P_{34} P+P P_{34} P\right) \psi_{A} \\
& +(1+P+\widetilde{P}+P \widetilde{P}) \psi_{B} .
\end{aligned}
$$

In order to simplify Eq. (A1), we consider a term with an arbitrary permutation operator $X$ acting on $\psi_{A}$. Inserting the $S$-wave projection operator, Eq. (16), we can write

$$
\begin{aligned}
& \frac{1}{3}\left\langle u_{1} u_{2} u_{3}\left|\left(1+G_{0} t_{12}\right) G_{0} V_{3} X\right| \psi_{A}\right\rangle=\frac{\lambda_{3}}{3}(4 \pi)^{2} G_{0}\left(u_{1}, u_{2}, u_{3}\right) \\
& \quad \times\left[\int d u_{1}^{\prime} u_{1}^{\prime 2} d u_{2}^{\prime} u_{2}^{\prime 2} \mathcal{D} u^{\prime \prime} u^{\prime \prime 2} \xi\left(u_{1}, u_{2}\right) \xi\left(u_{1}^{\prime}, u_{2}^{\prime}\right)\right. \\
& \quad \times\left\langle u_{1}^{\prime} u_{2}^{\prime} u_{3}|X| u_{1}^{\prime \prime} u_{2}^{\prime \prime} u_{3}^{\prime \prime}\right\rangle\left\langle u_{1}^{\prime \prime} u_{2}^{\prime \prime} u_{3}^{\prime \prime} \mid \psi_{A}\right\rangle \\
& +4 \pi \int d u_{1}^{\prime} u_{1}^{\prime 2} d u_{1}^{\prime \prime} u_{1}^{\prime \prime 2} d u_{2}^{\prime \prime} u_{2}^{\prime \prime 2} \mathcal{D} u^{\prime \prime \prime} u^{\prime \prime \prime 2} g\left(u_{1}\right) \\
& \quad \times \tau\left(E-\frac{3}{4} u_{2}^{2}-\frac{2}{3} u_{3}^{2}\right) g\left(u_{1}^{\prime}\right) G_{0}\left(u_{1}^{\prime}, u_{2}, u_{3}\right) \xi\left(u_{1}^{\prime}, u_{2}\right) \xi\left(u_{1}^{\prime \prime}, u_{2}^{\prime \prime}\right) \\
& \left.\quad \times\left\langle u_{1}^{\prime \prime} u_{2}^{\prime \prime} u_{3}|X| u_{1}^{\prime \prime \prime} u_{2}^{\prime \prime \prime} u_{3}^{\prime \prime \prime}\right\rangle\left\langle u_{1}^{\prime \prime \prime} u_{2}^{\prime \prime \prime} u_{3}^{\prime \prime \prime} \mid \psi_{A}\right\rangle\right],
\end{aligned}
$$

where the factors of $4 \pi$ and $(4 \pi)^{2}$ arise from the $S$-wave projection of the two-body $t$-matrix and three-body potential, respectively. This expression can be rewritten as

$$
\begin{aligned}
\frac{1}{3}\left\langle u_{1} u_{2} u_{3}\left|\left(1+G_{0} t_{12}\right) G_{0} V_{3} X\right| \psi_{A}\right\rangle \\
=\frac{\lambda_{3}}{3}(4 \pi)^{2} G_{0}\left(u_{1}, u_{2}, u_{3}\right) \\
\quad \times\left[\xi\left(u_{1}, u_{2}\right)+4 \pi g\left(u_{1}\right) \tau\left(E-\frac{3}{4} u_{2}^{2}-\frac{2}{3} u_{3}^{2}\right) \mathcal{I}\left(u_{2}, u_{3}\right)\right] \\
\quad \times \int d u_{1}^{\prime} u_{1}^{\prime 2} d u_{2}^{\prime} u_{2}^{\prime 2} \mathcal{D} u^{\prime \prime} u^{\prime \prime 2} \xi\left(u_{1}^{\prime}, u_{2}^{\prime}\right)\left\langle u_{1}^{\prime} u_{2}^{\prime} u_{3}|X| u_{1}^{\prime \prime} u_{2}^{\prime \prime} u_{3}^{\prime \prime}\right\rangle \\
\quad \times\left\langle u_{1}^{\prime \prime} u_{2}^{\prime \prime} u_{3}^{\prime \prime} \mid \psi_{A}\right\rangle \\
=\frac{\lambda_{3}}{3}(4 \pi)^{2} G_{0}\left(u_{1}, u_{2}, u_{3}\right)\left[\xi\left(u_{1}, u_{2}\right)+4 \pi g\left(u_{1}\right)\right. \\
\left.\quad \times \tau\left(E-\frac{3}{4} u_{2}^{2}-\frac{2}{3} u_{3}^{2}\right) \mathcal{I}\left(u_{2}, u_{3}\right)\right] K_{X}^{(A)}\left(u_{3}\right),
\end{aligned}
$$

where we have defined the quantities

$$
\mathcal{I}\left(u_{1}, u_{2}\right)=\int d u_{1}^{\prime} u_{1}^{\prime 2} G_{0}\left(u_{1}^{\prime}, u_{2}, u_{3}\right) g\left(u_{1}^{\prime}\right) \xi\left(u_{1}^{\prime}, u_{2}\right),
$$

and

$$
K_{X}^{(A)}\left(u_{3}\right)=\int d u_{1}^{\prime} u_{1}^{\prime 2} d u_{2}^{\prime} u_{2}^{\prime 2} \mathcal{D} u^{\prime \prime} u^{\prime \prime 2} \xi\left(u_{1}^{\prime}, u_{2}^{\prime}\right)\left\langle u_{1}^{\prime} u_{2}^{\prime} u_{3}|X| u_{1}^{\prime \prime} u_{2}^{\prime \prime} u_{3}^{\prime \prime}\right\rangle
$$$$
\times\left\langle u_{1}^{\prime \prime} u_{2}^{\prime \prime} u_{3}^{\prime \prime} \mid \psi_{A}\right\rangle .
$$

An analogous expression $K_{X}^{(B)}$ can be derived for permutation operators which act on the second Yakubovsky component $\psi_{B}$.

We proceed by giving the analytical expressions for the $K_{X}^{(A)}$ and $K_{X}^{(B)}$ which appear in the computation of the threebody force term. There are six combinations of permutation operators acting on $\psi_{A}$ :

$$
\begin{aligned}
& K_{1}^{(A)}=\int d u_{1}^{\prime} u_{1}^{\prime 2} d u_{2}^{\prime} u_{2}^{\prime 2} \xi\left(u_{1}^{\prime}, u_{2}^{\prime}\right)\left\langle u_{1}^{\prime} u_{2}^{\prime} u_{3} \mid \psi_{A}\right\rangle, \\
& K_{P_{34}}^{(A)}=\frac{1}{2} \int d u_{1}^{\prime} u_{1}^{\prime 2} d u_{2}^{\prime} u_{2}^{\prime 2} \xi\left(u_{1}^{\prime}, u_{2}^{\prime}\right) \\
& \times \int_{-1}^{1} d x^{\prime}\left\langle u_{1}^{\prime} \widetilde{u}_{2}\left(u_{2}^{\prime}, u_{3}\right) \widetilde{u}_{3}\left(u_{2}^{\prime}, u_{3}\right) \mid \psi_{A}\right\rangle \\
& K_{P P_{34}}^{(A)}=\frac{1}{2} \int d u_{2}^{\prime} u_{2}^{\prime 2} d u_{2}^{\prime \prime} u_{2}^{\prime \prime 2} \int_{-1}^{1} d x \int_{-1}^{1} d x^{\prime} \xi\left(\pi\left(u_{2}^{\prime}, u_{2}^{\prime \prime}\right), u_{2}^{\prime}\right) \\
& \times\left\langle\pi\left(u_{2}^{\prime \prime}, u_{2}^{\prime}\right) \tilde{u}_{2}\left(u_{2}^{\prime \prime}, u_{3}\right) \tilde{u}_{3}\left(u_{2}^{\prime \prime}, u_{3}\right) \mid \psi_{A}\right\rangle, \\
& K_{P}^{(A)}=\int d u_{2}^{\prime} u_{2}^{\prime 2} d u_{2}^{\prime \prime} u_{2}^{\prime \prime 2} \int_{-1}^{1} d x \xi\left(\pi\left(u_{2}^{\prime}, u_{2}^{\prime \prime}\right), u_{2}^{\prime}\right) \\
& \times\left\langle\pi\left(u_{2}^{\prime \prime}, u_{2}^{\prime}\right) u_{2}^{\prime \prime} u_{3} \mid \psi_{A}\right\rangle, \\
& K_{P_{34} P}^{(A)}=\frac{1}{2} \int d u_{2}^{\prime} u_{2}^{\prime 2} d u_{2}^{\prime \prime} u_{2}^{\prime \prime 2} \int_{-1}^{1} d x \\
& \times \int_{-1}^{1} d x^{\prime} \xi\left(\pi\left(\tilde{u}_{2}\left(u_{2}^{\prime}, u_{3}\right), u_{2}^{\prime \prime}\right), u_{2}^{\prime}\right) \\
& \times\left\langle\pi\left(u_{2}^{\prime \prime}, \tilde{u}_{2}\left(u_{2}^{\prime}, u_{3}\right)\right) u_{2}^{\prime \prime} \widetilde{u}_{3}\left(u_{2}^{\prime}, u_{3}\right) \mid \psi_{A}\right\rangle, \\
& K_{P P_{34} P}^{(A)}=\frac{1}{2} \int d u_{2}^{\prime} u_{2}^{\prime 2} d u_{2}^{\prime \prime} u_{2}^{\prime \prime 2} \int_{-1}^{1} d x \int_{-1}^{1} d x^{\prime} \int_{-1}^{1} d x^{\prime \prime} \\
& \times \xi\left(\pi\left(u_{2}^{\prime}, u_{2}^{\prime \prime}\right), u_{2}^{\prime}\right)\left\langle\hat{u}_{1}\left[\pi\left(u_{2}^{\prime \prime}, u_{2}^{\prime}\right), \widetilde{u}_{2}\left(u_{2}^{\prime \prime}, u_{3}\right)\right]\right. \\
& \times \hat{u}_{2}\left[\pi\left(u_{2}^{\prime \prime}, u_{2}^{\prime}\right), \widetilde{u}_{2}\left(u_{2}^{\prime \prime}, u_{3}\right)\right] \widetilde{u}_{3}\left(u_{2}^{\prime \prime}, u_{3}\right)\left|\psi_{A}\right\rangle \text {, }
\end{aligned}
$$

and four combinations of operators acting on $\psi_{B}$ :

$$
\begin{aligned}
K_{1}^{(B)}= & \frac{1}{2} \int d u_{1}^{\prime} u_{1}^{\prime 2} d u_{2}^{\prime} u_{2}^{\prime 2} \int_{-1}^{1} d x^{\prime} \xi\left(u_{1}^{\prime}, u_{2}^{\prime}\right) \\
& \times\left\langle u_{1}^{\prime} \widetilde{v}_{2}\left(u_{2}^{\prime}, u_{2}\right) \widetilde{v}_{3}\left(u_{2}^{\prime}, u_{3}\right) \mid \psi_{B}\right\rangle,
\end{aligned}
$$

$$
K_{P}^{(B)}=\frac{1}{2} \int d u_{2}^{\prime} u_{2}^{\prime 2} d u_{2}^{\prime \prime} u_{2}^{\prime \prime 2} \int_{-1}^{1} d x \int_{-1}^{1} d x^{\prime} \xi\left(\pi\left(u_{2}^{\prime}, u_{2}^{\prime \prime}\right), u_{2}^{\prime}\right)
$$$$
\times\left\langle\pi\left(u_{2}^{\prime \prime}, u_{2}^{\prime}\right) \widetilde{v}_{2}\left(u_{2}^{\prime \prime}, u_{3}\right) \widetilde{v}_{3}\left(u_{2}^{\prime \prime}, u_{3}\right) \mid \psi_{B}\right\rangle,
$$ 


$$
\begin{aligned}
& K_{\widetilde{P}}^{(B)}=\frac{1}{2} \int d u_{1}^{\prime} u_{1}^{\prime 2} d u_{2}^{\prime} u_{2}^{\prime 2} \int_{-1}^{1} d x^{\prime} \xi\left(u_{1}^{\prime}, u_{2}^{\prime}\right) \\
& \times\left\langle\widetilde{v}_{3}\left(u_{2}^{\prime}, u_{3}\right) \widetilde{v}_{2}\left(u_{2}^{\prime}, u_{3}\right) u_{1}^{\prime} \mid \psi_{B}\right\rangle, \\
& K_{P \widetilde{P}}^{(B)}=\frac{1}{2} \int d u_{2}^{\prime} u_{2}^{\prime 2} d u_{2}^{\prime \prime} u_{2}^{\prime \prime 2} \int_{-1}^{1} d x \int_{-1}^{1} d x^{\prime} \xi\left(\pi\left(u_{2}^{\prime}, u_{2}^{\prime \prime}\right), u_{2}^{\prime}\right) \\
& \times\left\langle\widetilde{v}_{3}\left(u_{2}^{\prime \prime}, u_{3}\right) \widetilde{v}_{2}\left(u_{2}^{\prime \prime}, u_{3}\right) \pi\left(u_{2}^{\prime \prime}, u_{2}^{\prime}\right) \mid \psi_{B}\right\rangle, \\
& \hat{u}_{1}\left(u_{1}, u_{2}\right)=\sqrt{\frac{1}{4} u_{1}^{2}+\frac{9}{16} u_{2}^{3}+\frac{3}{4} u_{1} u_{2} x^{\prime \prime},} \\
& \hat{u}_{2}\left(u_{1}, u_{2}\right)=\sqrt{u_{1}^{2}+\frac{1}{4} u_{2}^{2}-u_{1} u_{2} x^{\prime \prime}} .
\end{aligned}
$$

[1] S. R. Beane, P. F. Bedaque, W. C. Haxton, D. R. Phillips, and M. J. Savage, At the Frontier of Particle Physics edited by M. Shifman (World Scientific, Singapore, 2001), Vol. 1.

[2] P. F. Bedaque and U. van Kolck, Annu. Rev. Nucl. Part. Sci. 52, 339 (2002).

[3] H.-W. Hammer, Nucl. Phys. A 737, 275 (2004).

[4] J. O. Andersen, Rev. Mod. Phys. 76, 599 (2004).

[5] D. B. Kaplan, M. J. Savage, and M. B. Wise, Phys. Lett. B 424, 390 (1998).

[6] U. van Kolck, Nucl. Phys. A 645, 273 (1999).

[7] P. F. Bedaque, H. W. Hammer, and U. van Kolck, Phys. Rev. Lett. 82, 463 (1999).

[8] P. F. Bedaque, H.-W. Hammer, and U. van Kolck, Nucl. Phys. A 646, 444 (1999).

[9] V. Efimov, Yad. Fiz. 12, 1080 (1970); [Sov. J. Nucl. Phys. 12, 589 (1971)].

[10] V. Efimov, Yad. Fiz. 29, 1058 (1979); [Sov. J. Nucl. Phys. 29, 546 (1979)].

[11] G. P. Lepage, e-print nucl-th/9706029.

[12] K. G. Wilson, talk presented at the INT program on "Effective Field Theory and Effective Interactions," Institute for Nuclear Theory, Seattle, 2000 (unpublished).

[13] R. F. J. Mohr, e-print nucl-th/0306086.

[14] J. A. Tjon, Phys. Lett. 56, 217 (1975).

[15] B. F. Gibson and D. R. Lehman, Phys. Rev. C 14, 685 (1976).

[16] A. C. Fonseca and P. E. Shanley, Phys. Rev. C 14, 1343 (1976).

[17] S. Nakaichi-Maeda and T. K. Lim, Phys. Rev. A 28, 692 (1983).

[18] J. A. Tjon, in Few-body Systems and Nuclear Forces II, edited by H. Ziegel et al. (Springer, Berlin, 1978).

[19] T. K. Lim, Nucl. Phys. A 416, 491 (1984).

[20] M. Lewerenz, J. Chem. Phys. 106, 4596 (1997).

[21] D. Blume and C. H. Greene, J. Chem. Phys. 112, 8053 (2000).

[22] I. N. Filikhin, S. L. Yakovlev, V. A. Roudnev, and B. Vlahovic, J. Phys. B 35, 501 (2002).

[23] K. B. Whaley, Int. Rev. Phys. Chem. 13, 41 (1994).

[24] H. Kamada, Phys. Rev. C 64, 044001 (2001).

[25] O. A. Yakubovsky, Yad. Fiz. 5, 1312 (1967); [Sov. J. Nucl. Phys. 5, 937 (1967)].

[26] E. Braaten and H. W. Hammer, Phys. Rev. A 67, 042706 (2003).

[27] E. Braaten, H. W. Hammer, and M. Kusunoki, Phys. Rev. A 67, 022505 (2003).
[28] S. K. Adhikari, T. Frederico, and I. D. Goldman, Phys. Rev. Lett. 74, 487 (1995).

[29] R. D. Amado and F. C. Greenwood, Phys. Rev. D 7, 2517 (1973).

[30] A. Nogga, H. Kamada, and W. Glöckle, Phys. Rev. Lett. 85, 944 (2000).

[31] E. Epelbaum, A. Nogga, W. Glöckle, H. Kamada, U.-G. Meißner, and H. Witala, Phys. Rev. C 66, 064001 (2002).

[32] J. V. Steele and R. J. Furnstahl, Nucl. Phys. A 637, 46 (1998).

[33] D. Espriu and J. Matias, Nucl. Phys. B 418, 494 (1994).

[34] H. W. Hammer and T. Mehen, Phys. Lett. B 516, 353 (2001).

[35] P. F. Bedaque, G. Rupak, H. W. Griesshammer, and H. W. Hammer, Nucl. Phys. A 714, 589 (2003).

[36] I. R. Afnan and D. R. Phillips, Phys. Rev. C 69, 034010 (2004).

[37] E. W. Schmid and H. Ziegelmann, The Quantum Mechanical Three-Body Problem (Vieweg, Braunschweig, 1971).

[38] L. D. Faddeev, Zh. Eksp. Teor. Fiz. 39, 1459 (1960); [Sov. Phys. JETP 12, 1014 (1961)].

[39] W. Meier and W. Glöckle, Phys. Rev. C 28, 1807 (1983).

[40] W. Glöckle, The Quantum Mechanical Few-Body Problem (Springer, New York, 1983).

[41] W. Glöckle and H. Kamada, Nucl. Phys. A 560, 541 (1993).

[42] R. E. Grisenti et al., Phys. Rev. Lett. 85, 2284 (2000).

[43] W. Schöllkopf and J. P. Toennies, J. Chem. Phys. 104, 1155 (1996).

[44] L. W. Bruch, W. Schöllkopf, and J. P. Toennies, J. Chem. Phys. 117, 1544 (2002).

[45] E. Nielsen, D. V. Fedorov, and A. S. Jensen, J. Phys. B 31, 4085 (1998).

[46] V. Roudnev and S. Yakovlev, Chem. Phys. Lett. 328, 97 (2000).

[47] A. K. Motovilov, W. Sandhas, S. A. Sofianos, and E. A. Kolganova, Eur. Phys. J. D 13, 33 (2001).

[48] P. Barletta and A. Kievsky, Phys. Rev. A 64, 042514 (2001).

[49] R. A. Aziz and M. J. Slaman, J. Chem. Phys. 94, 8047 (1991).

[50] T. Frederico, L. Tomio, A. Delfino, and A. E. A. Amorim, Phys. Rev. A 60, R9 (1999).

[51] A. Delfino, T. Frederico, and L. Tomio, Few-Body Syst. 28, 259 (2000).

[52] D. S. Petrov, C. Salomon, and G. V. Shlyapnikov, Phys. Rev. Lett. 93, 090404 (2004).

[53] F. M. Marqués et al., Phys. Rev. C 65, 044006 (2002). 